\title{
Inhibition of autophagy enhances synergistic effects of Salidroside and anti-tumor agents against colorectal cancer
}

\author{
Hai $\mathrm{Li}^{1 *}$ and Chen Chen ${ }^{2}$
}

\begin{abstract}
Background: Various plant extracts have been suggested to be used as auxiliary agents in chemotherapy considering their anti-proliferative effect on cancer cells. However, recent reports reveal that plant extracts may function as inducers of autophagy of cancer cells. In general, autophagy confers survival advantage for cells responding to stress conditions, thus representing an important mechanism for chemo-resistance. This study was aimed to investigate the effectiveness of combined use of Salidroside (Sal, a phenylpropanoid glycosides from Rhodiola rosea $\mathrm{L}$ ) with anti-tumor agents against colorectal cancer (CRC) cells, and moreover to evaluate the potential role of autophagy in the combined therapy.

Methods: CRC cells, HCT-116, were incubated with Sal alone or in combination with conventional chemotherapy agents including oxaliplatin (OXA), 5-fluorouracil (5-FU) and Doxorubicin (ADM). Cell proliferative characteristics were evaluated by cell viability and apoptosis rate. The protein expression was assessed by Immunofluorescent and Western blot assays.

Results: Sal, alone or in combination with anti-tumor agents, increased expression of autophagic biomarkers, including LC3B and Becline-1, suggesting an autophagy induction. Except for the up-regulation of p-AMPK, p-mTOR, p-NF-kB (p65), TGF- $\beta$, p-JAK2 and p-STAT3 were down-regulated by Sal. Because autophagy is positively correlated with the activation of AMPK/mTOR, NF-KB, TGF $\beta 1$ and JAK2/STAT3 cascades, the autophagy induced by Sal may associate with AMPK activation. Indeed, blockage of AMPK signaling via Compound C or AMPK knockdown inhibited the autophagy. The blockage of AMPK signaling or a direct inhibition of autophagy via 3-MA increased effectiveness of combined use of Sal with anti-tumor agents against CRC.
\end{abstract}

Conclusions: Inhibition of autophagy enhances synergistic effects of Sal and anti-tumor agents against colorectal cancer. This study provides experimental evidence and theoretical reference for improvement of a novel chemotherapy treatment protocol.

Keywords: Salidroside, Autophagy, AMPK signaling, Anti-tumor agents, Colorectal cancer

\section{Background}

Although recent advances in diagnostic, surgical, and therapeutic techniques, colorectal cancer (CRC) causes high morbidity and mortality with approximately $62.7 \%$ of the 5-year survival rate worldwide [1,2]. Chemotherapy remains one of the most common treatments for colon cancer, specifically for patients with the recurrent

\footnotetext{
*Correspondence: lihaiges@hust.edu.cn

'Department of Geriatrics, Tongji Hospital, Tongji Medical College, Huazhong

University of Science and Technology, Wuhan, China

Full list of author information is available at the end of the article
}

and metastatic diseases, to prolong survival time [1]. Currently, researchers attempt to add auxiliary agents to create additive or synergistic effects on standard treatment regimens. It's long been known that numerous plant extracts possess potently anti-proliferative and pro-apoptotic effects against diverse cancer cells, and the intake is associated with reduced incidence and slow development of cancers [3]. However the effectiveness of using chemotherapeutic agents and plant extracts in combination is contradictory in cancer therapy in different studies. Herbal agent MB-6, which is derived from 
fermented soybean, green tea, grape seed and curcumin extracts, increase the effectiveness 5-fluoracil-based chemotherapy in CRC [4]. In addition, herb extract Tanshinone IIA improves the sensibility of chemotherapeutics for non-small cell lung cancer with fewer side effects, which may be a promising sensitizers for chemotherapy drugs [5]. However, evidence is increasing that some botanical extracts induce generation of autophagy of cancer cells, which impairs the toxicity of chemotherapeutic agents [6].

Autophagy is an adaptive response to nutrient deprivation and environmental stimulus, which can occur in both normal and cancer cells. It involves the lysosomal degradation of cellular components such as misfolded proteins or damaged organelles, to remove dysfunctional cytoplasmic constituents and recycle basic molecular building blocks [7]. The process not only maintains metabolic homeostasis, but also protects against cells death under adverse conditions. At present, autophagy has become an important anticancer target in chemotherapy, because autophagy is associated with increased chemo-resistance of cancer cells, responsible for consequent therapeutic failure [8]. In spite of their anti-proliferative effect, many plant extracts trigger the generation of autophagy [7, 9]. Autophagy is regulated by complicated signaling pathways of AMPK/mTOR, NF$\kappa \mathrm{B}, \mathrm{TGF}-\beta, \mathrm{JAK} 2 / \mathrm{STAT} 3$ and so on [10-13]. Currently, the mechanism underlying the autophagy induced by botanical extracts is not well-understood.

Salidroside ( $\mathrm{Sal}$ ) is one of the major phenylpropanoid glycosides in Rhodiola rosea $L$, a medicinally important plant mainly found in the alpine area of China [14]. Previous reports have manifested that Sal administration inhibits the growth of human cancers, including CRC, breast cancer, lung cancer and renal cell carcinoma in vitro and in vivo [14-16]. This study was aimed to investigate the effectiveness of combined use of Sal with antitumor agents against CRC cells and moreover to evaluate the potential role of autophagy in the combined therapy.

\section{Methods}

\section{Cell culture and treatment}

CRC cell line was obtained from the American Type Culture Collection (ATCC CCL-247 $7^{\mathrm{rm}}$, Manassas, VA, USA). HCT-116 cells were cultured in Dulbecco's modified Eagle's medium (Gibco-BRL, Grand Island, NY, USA) supplemented with $10 \%$ fetal bovine serum (Invitrogen, Life Technologies, Carlsbad, CA, USA) in a $37{ }^{\circ} \mathrm{C}$ incubator with $5 \% \mathrm{CO}_{2}$.

Sal was purchased from Sigma-Aldrich (No. 4386625MG; St Louis, MO, USA). HCT-116 cells in the logarithmic phase were treated with $\operatorname{Sal}(0,0.5,1$ or $2 \mu \mathrm{g} / \mathrm{mL}$ ) for different time periods to characterize the growth inhibitory effects. Besides, HCT-116 cells were subjected to antitumor drugs including oxaliplatin (OXA; NO. O9512), 5-fluorouracil (5-FU; NO. 04541) and Doxorubicin (ADM; NO. D1515; Sigma-Aldrich) to determine their IC25 values. Furthermore, HCT-116 cells were treated with Sal and antitumor drugs together to investigate their synergistic effects. Compound $\mathrm{C}$ and 3-Methyladenine (3-MA) were purchased from Selleck (Houston, Texas, USA). Their were added to cells to block AMPK signaling and autophagy, respectively.

\section{Cell viability assay}

HCT-116 cell viability was evaluated using CCK-8 Detection Kit (Beyotime Institute of Biotechnology, Shanghai, China). HCT-116 cells were seeded into 96well plates and subjected to indicated treatments. The cells were further maintained in $90 \mu \mathrm{l}$ of culture medium plus $10 \mu \mathrm{l}$ CCK-8 reagents per well for $1 \mathrm{~h}$. The optical density at $490 \mathrm{~nm}$ (OD $490 \mathrm{~nm}$ ) in each well was determined by an enzyme immunoassay analyzer (BioTek ELX-800; Winooski, VT, USA).

\section{Cell apoptosis rate assay}

The cell apoptosis rate was assessed using an Annexin V-FITC/PI Apoptosis Detection Kit (Beyotime Institute of Biotechnology) according to the manufacturer's instructions. The HCT-116 cells were double-stained with Annexin V-FITC and propidium iodide in the dark, and then analyzed with a dual laser flow cytometer (Becton Dickinson, San Jose, CA, USA).

\section{Immunofluorescent (IF) assay}

The HCT-116 cells were fixed with $4 \%$ paraformaldehyde for $15 \mathrm{~min}$ and blocked with PBS containing $0.3 \%$ Triton $\mathrm{X}-100 / 5 \%$ BSA $(w / v)$ for $1 \mathrm{~h}$ at room temperature, before the incubation with antibody specific for LC3B (1:500, ab48394; Abcam, Cambridge, UK). Incubation with the secondary fluorescent-labeled antibody (Alexa Fluor 488, Invitrogen) was performed in the dark prior to the microscopic analysis (LeicaTCS-SP5 microscopy, Leica instrument co., LTD, Beijing, China).

\section{Western blotting}

Total cell extracts were prepared using ice-cold lysis buffer (Sigma-Aldrich) containing protease inhibitor cocktail (Sigma-Aldrich). Proteins (20 $\mu \mathrm{g} /$ lane) were separated by SDS-polyacrylamide gel electrophoresis (10-15\% gels) and transferred onto nitrocellulose membranes (Sigma-Aldrich). Membranes were blocked in 5\% non-fat milk in TBS/0.1\% Tween 20 for $2 \mathrm{~h}$ prior to immunoblotting overnight with antibodies against LC3B (1:500, ab48394; Abcam), phospho (p)-AMPKa (1:500; \#2535, Cell Signaling Technology, Inc., Shanghai, China), p-mTOR (1:1000; \#2971, Cell Signaling Technology), p- 
NF-кB (p65) (1:500; sc-166,748, Santa Cruz Biotechnology, Inc., Dallas, TX, USA), TGF 11 (1:1000; ab31013, Abcam), p-JAK2 (1:1000; ab195055, Abcam), p-STAT3 (1:800; ab30647, Abcam), Beclin-1(1:500; ab55878, Abcam) and $\beta$ actin (1:1000, Santa Cruz Biotechnologies). Incubation with the secondary fluorescent-labeled antibody (Alexa Fluor 488) was performed for $2 \mathrm{~h}$ at room temperature in the dark. The proteins were visualized by enhanced chemiluminescence (Amersham Bio-sciences, NJ, USA).

\section{The knockdown of AMPKa}

Small interference RNA (siRNA) targeting AMPK $\alpha$ (siRNA-AMPK $\alpha$ ) was synthesized by GenePharma Co., Ltd. (Shanghai, China). The double-stranded sequences were: 5'-AUUCAUGUGUGCAUCAAGCTT-3'; 5'GCUUGAUGCACACAUGAAUTT-3'. SiRNA-AMPK $\alpha$ was transfected into HCT-116 cells using Lipofecta$\operatorname{mine}^{\mathrm{rm}} 2000$ (Invitrogen Life Technologies), according to the manufacturer's instructions. Transfection efficiency was routinely 85 to $90 \%$, as determined by transfection of enhanced green fluorescent protein reporter plasmid.

\section{Statistical analysis}

For statistical analysis the Student's t test was applied (SPSS13.0 software; Chicago, IL, USA). The results are presented as the mean \pm S.E. of three independent experiments. $p$ values of $<0.05$ was considered as significant and marked with an asterisk.

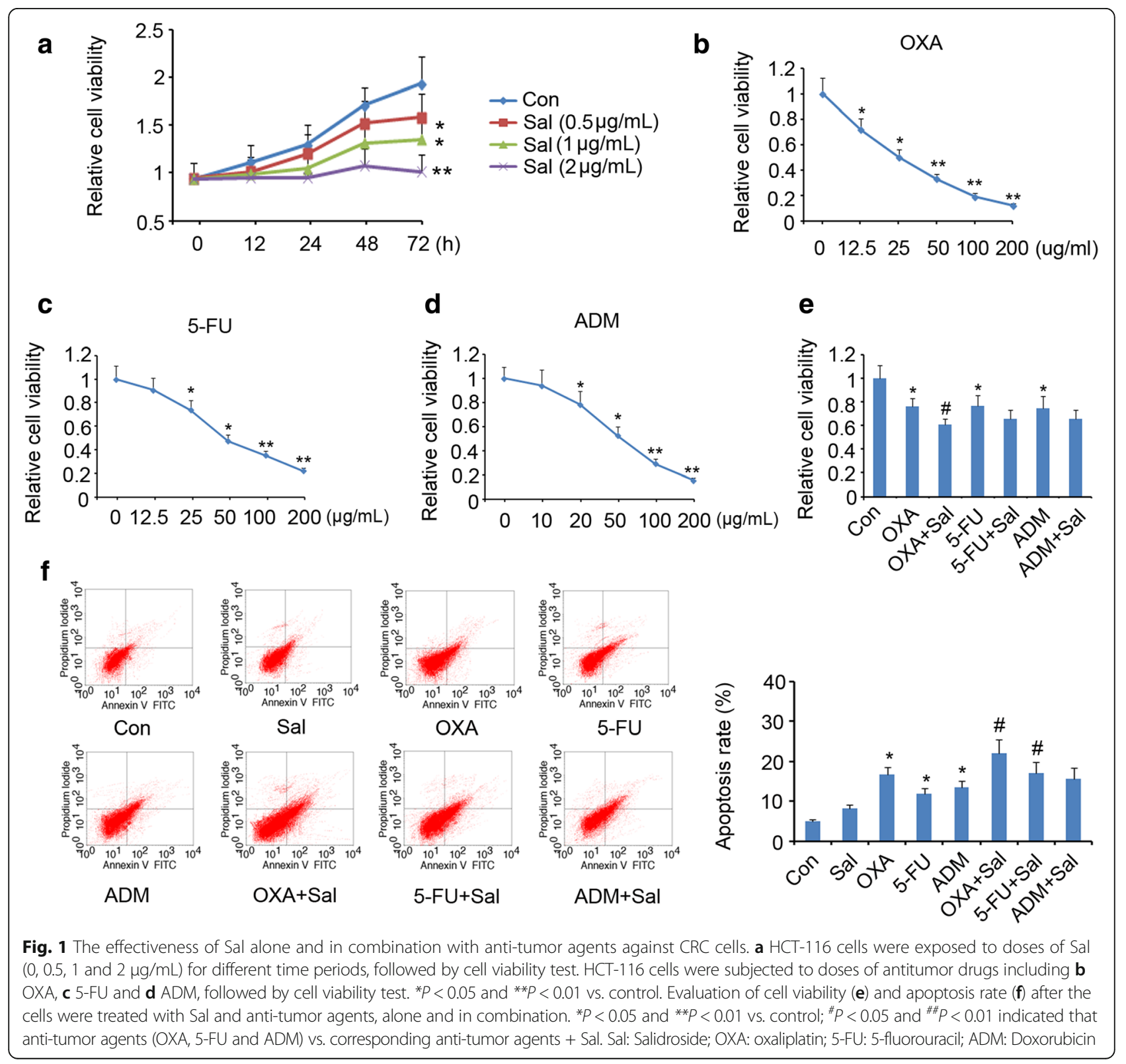




\section{Results}

Effectiveness of combined use of Sal with anti-tumor drugs against HCT-116 cells

This study initially characterized the growth inhibitory effect of Sal on HCT-116 cells. HCT-116 cells were exposed to $\mathrm{Sal}(0,0.5,1$ or $2 \mu \mathrm{g} / \mathrm{mL})$ for different time periods. As shown in Fig. 1a, adding 0.5 and $1 \mu \mathrm{g} / \mathrm{mL}$ Sal for $72 \mathrm{~h}$ inhibited viability of HCT-116 cells $(p<0.05) .2 \mu \mathrm{g} / \mathrm{mL}$ Sal caused more effective inhibition of the cell viability than 0.5 and $1 \mu \mathrm{g} / \mathrm{mL}$ Sal $(p<0.01$ vs. control, at $72 \mathrm{~h})$.

HCT-116 cells were subjected to various concentrations of antitumor drugs including OXA, 5-FU and
ADM to determine their IC25 values. The growth curves of HCT-116 cells after exposing to OXA, 5-FU and ADM were shown in Fig. 1b, c and d respectively. HCT116 cell viability was decreased by $25 \%$ by $12.93 \mu \mathrm{g} / \mathrm{mL}$ OXA, $25.75 \mu \mathrm{g} / \mathrm{mL} 5$-FU or $19.68 \mu \mathrm{g} / \mathrm{mL}$ ADM.

Treatment with $1 \mu \mathrm{g} / \mathrm{mL}$ Sal and $12.93 \mu \mathrm{g} / \mathrm{mL}$ OXA in combination showed improved effect on the inhibition of the cell viability compared to treatment with $12.93 \mu \mathrm{g} / \mathrm{mL}$ OXA alone $(p<0.05$, Fig. 1e). But, inhibitory effects of 5 -FU $(25.75 \mu \mathrm{g} / \mathrm{mL})$ and ADM $(19.68 \mu \mathrm{g} / \mathrm{mL})$ were only moderately enhanced by $1 \mu \mathrm{g} / \mathrm{mL}$ Sal.

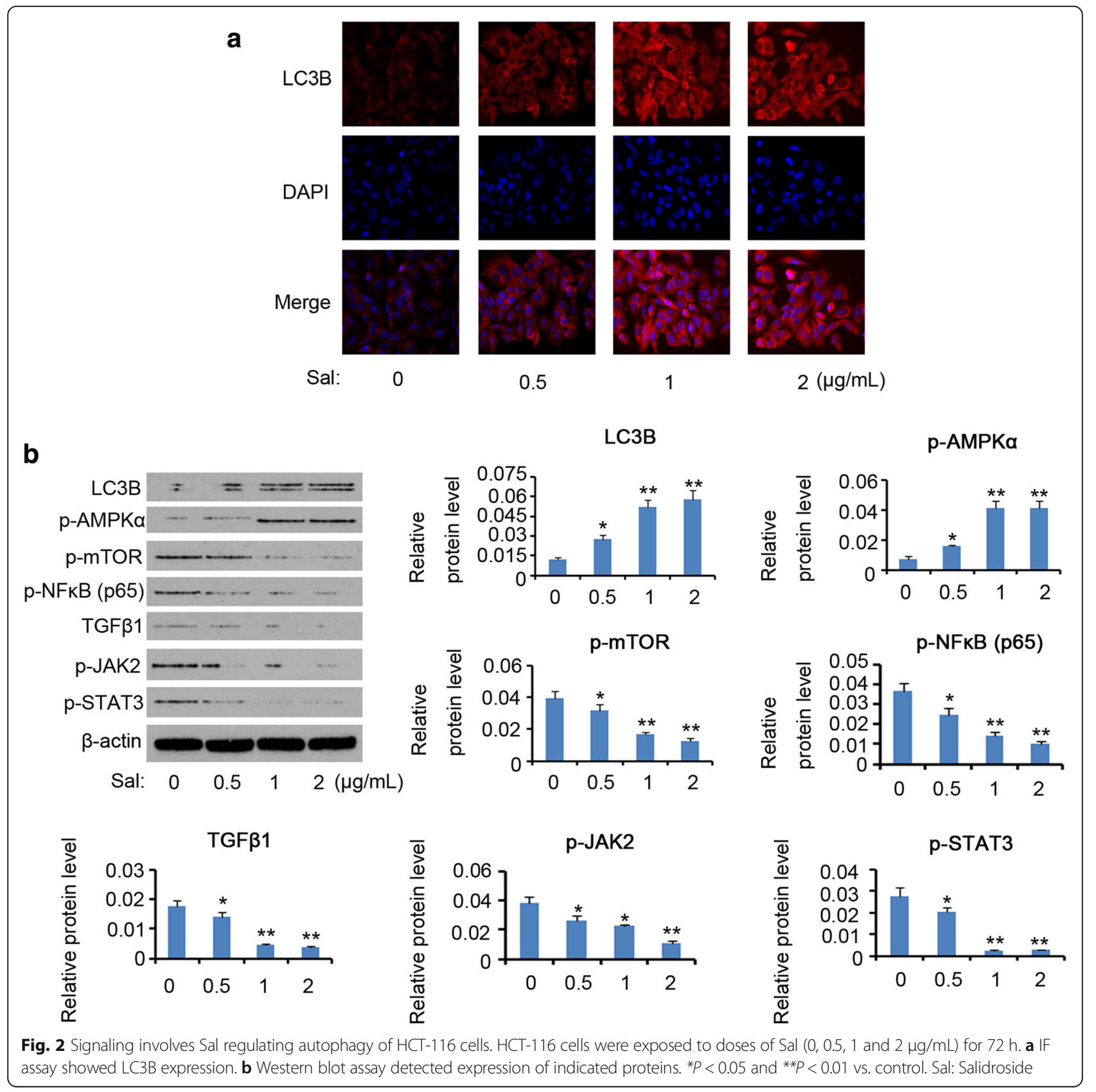


Apoptosis assay showed that treatment with $1 \mu \mathrm{g} / \mathrm{mL} \mathrm{Sal}$ for $72 \mathrm{~h}$ did not decrease apoptosis rate of HCT-116 cells significantly, but the apoptosis rate was reduced by OXA, 5FU and ADM at concentrations of $12.93 \mu \mathrm{g} / \mathrm{mL}$, $25.75 \mu \mathrm{g} / \mathrm{mL}$ and $19.68 \mu \mathrm{g} / \mathrm{mL}$, respectively $(p<0.05$, Fig. 1f). $1 \mu \mathrm{g} / \mathrm{mL}$ Sal conferred promoted effect on the apoptosis induced by OXA and 5-FU $(p<0.05)$, but only marginally increased the apoptosis induced by not by ADM.

\section{Sal promoted autophagy of HCT-116 cells through activating AMPK signaling}

To determine whether Sal promotes autophagy of HCT116 cells, we examined expression levels of autophagic biomarkers following the treatment with Sal. In IF assay, fluorescence intensity of LC3B (microtubule-associated protein 1 light chain 3B), a well-known autophagic biomarker, was increased by $\mathrm{Sal}$ in a dose-dependent manner (Fig. 2a). Western blot measurement further confirmed that Sal forced LC3B expression in HCT-116 cells (Fig. 2b). To get insight into the mechanism underlying the autophagy triggered by Sal, this study detected expression of signaling molecules that control autophagy process. Sal dose-dependently increased expression of p-AMPK, but reduced p-mTOR, p-NF-кB (p65), TGF $\beta 1$, $\mathrm{p}-\mathrm{JAK} 2$ and $\mathrm{p}-\mathrm{STAT} 3$ expression. Previous literature represents that autophagy associates activation of AMPK/mTOR, NF-kB, TGF $\beta 1$ and JAK2/STAT3 cascades [10-13], thus it was likely that AMPK/mTOR signaling mediates Sal-induced autophagy.

We further investigated the influence of combined use of Sal with anti-tumor agents on cell autophagy. $1 \mu \mathrm{g} / \mathrm{mL}$ Sal elevated expression of LC3B $(p<0.05)$ and Becline$1(p<0.05)$, as shown in Fig. 3. However, anti-tumor agents

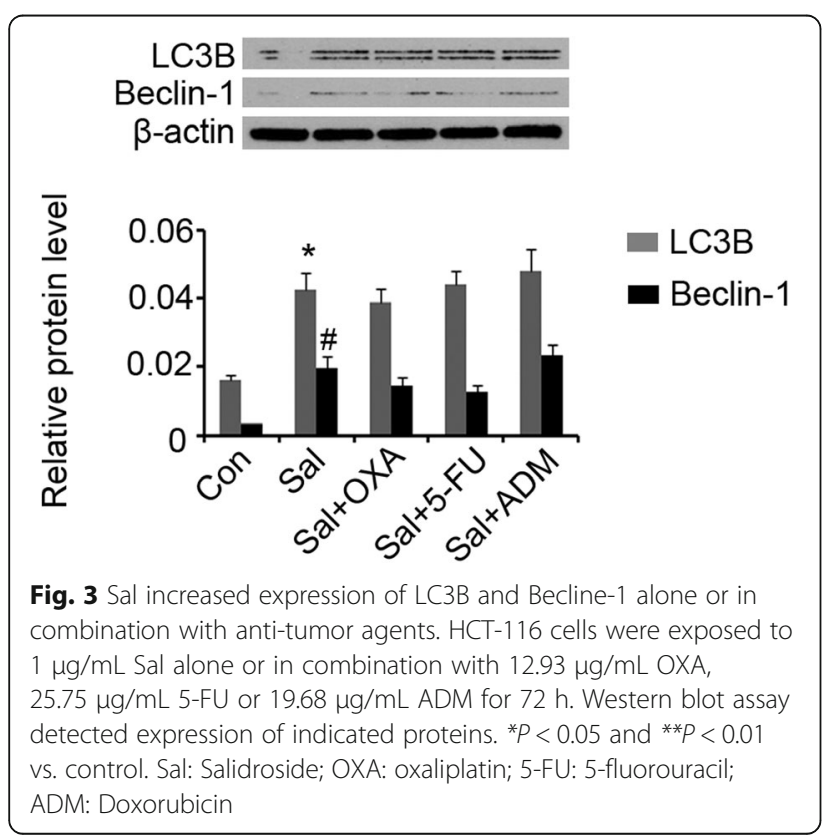

including OXA, 5-FU and ADM did not significantly up-regulated $\mathrm{LC} 3 \mathrm{~B}$ and Becline-1.

To determine the role of AMPK/mTOR signal in Sal-induced autophagy, this study blocked this signal through adding AMPK inhibitor (Compound $\mathrm{C}$ ) and knocking down AMPK via siRNA-AMPK $\alpha .10 \mu \mathrm{M}$ Compound $C$ reversed the up-regulation of $\mathrm{p}-\mathrm{AMPK}$ induced by $\mathrm{Sal}(p<0.05$ vs. control, Fig. $4 \mathrm{a})$. In addition, co-treatment of the Compound $\mathrm{C}$, Sal and anti-tumor agent (OXA, 5-FU or ADM) decreased p-AMPK protein level $(p<0.05$ vs. control). Compound $\mathrm{C}$ restored $\mathrm{p}-\mathrm{mTOR}$ level that was decreased by Sal. Sal increased LC3B expression ( $p<0.05$ vs. control), but treatment with Sal and Compound $\mathrm{C}$ in combination reversed LC3B expression ( $p<0.01$ vs. control). Adding OXA had modest effect on LC3B expression that was decreased by combined use of Sal and Compound C ( $p<0.01$ vs. control), whereas 5-FU and ADM to some degree restored LC3B expression $(p<0.05$ vs. control). Transfection with siRNA-AMPK $\alpha$ was performed for AMPK knockdown. The transfection efficiency was routinely determined by transfection of enhanced green fluorescent protein reporter plasmid (Fig. 4b). AMPK knockdown before the treatment with Sal suppressed the $\mathrm{p}$-AMPK and LC3B expression ( $p$ $<0.01$ vs. control, Fig. 4a) and restored the decreased pmTOR. 3-MA is commonly used to block the initiation of autophagy. 3-MA reversed the up-regulation of LC3B induced by Sal $(p<0.01$ vs. control), but conferred modest effects on $\mathrm{p}$-AMPK and p-mTOR protein levels.

The effectiveness of combined use of Sal with anti-tumor drugs was increased by inhibiting AMPK signal and autophagy

Compound C, AMPK knockdown and 3-MA marginally decreased viability of the HCT-116 cells treated with Sal (Fig. 5a). However, Compound C, AMPK knockdown and 3-MA dramatically reduced viability of the cells treated with Sal and anti-tumor drug (OXA, 5-FU or $\mathrm{ADM})$ in combination $(p<0.05$, Fig. $5 \mathrm{~b})$. Apoptosis rate of the HCT-116 cells treated with Sal was increased by AMPK knockdown $(p<0.05$, Fig. 5 c), but not by treatment with Compound $\mathrm{C}$ or 3-MA. Compound C, AMPK knockdown and 3-MA notably promoted apoptosis induced by combined use of Sal and anti-tumor drug (OXA, 5-FU or ADM) $(p<0.05$, Fig. $5 \mathrm{~d}-\mathrm{f})$.

\section{Discussion}

Various plant extracts have been suggested to be used as auxiliary agents in chemotherapy due to their antiproliferative and pro-apoptotic effects. However, combined use of chemotherapeutic agents and plant extracts is rather controversial in cancer therapy. That is because some plant extracts trigger the generation of autophagy of cancer cells while suppressing the proliferation $[7,9]$. 


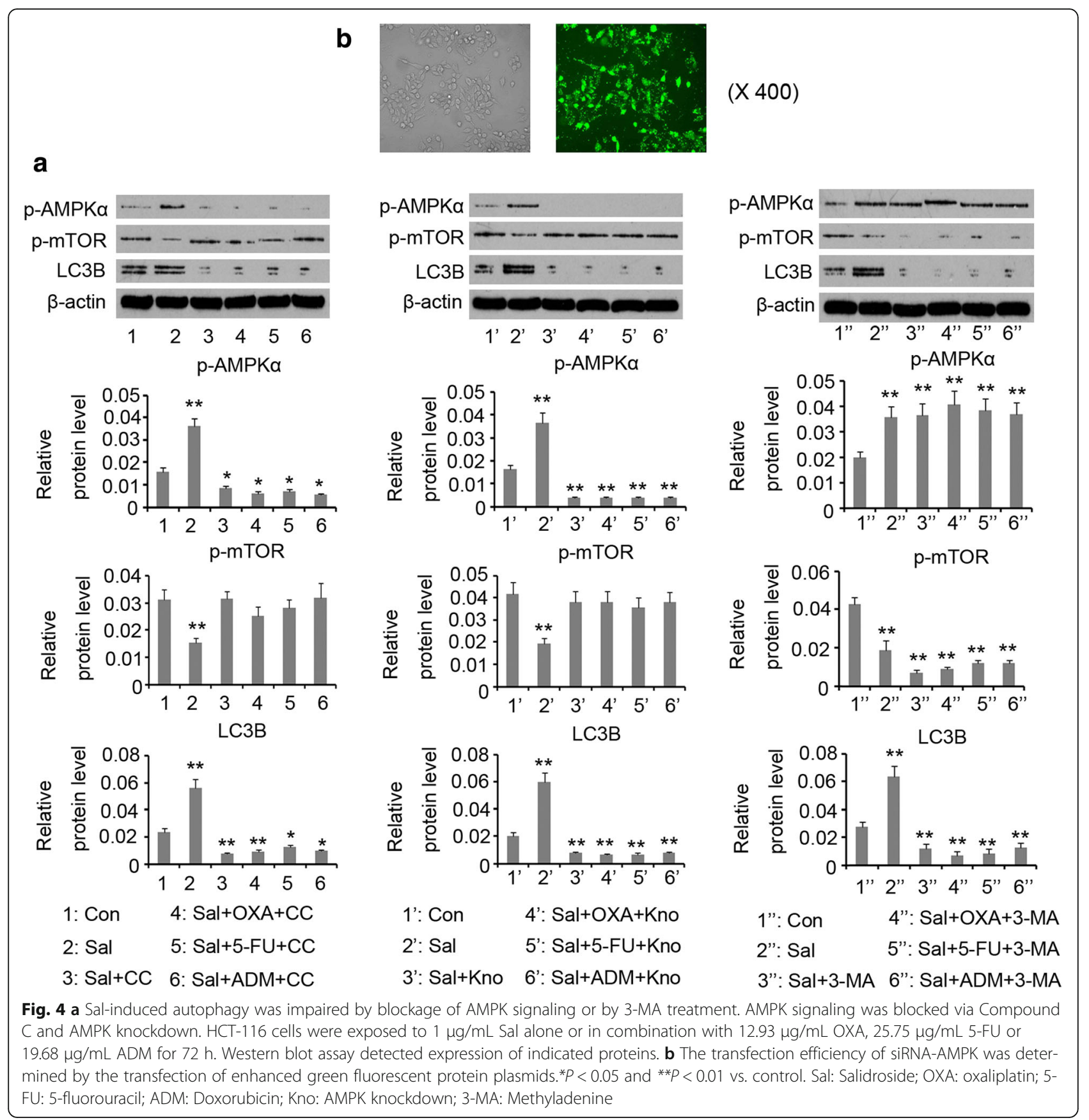

Autophagy confers protective effect against toxicity of anti-tumor agents, thus it, to some extent, inhibits apoptosis of cancerous cells and results in chemo-resistance [17]. Sal has been emerged as an important plant extracts for the treatments of cancer and age-related diseases [18]. Our data showed that Sal inhibited cell viability of CRC, but only exerted modest effect on the apoptosis at a moderate concentration $(1 \mu \mathrm{g} / \mathrm{mL})$. Sal showed synergistic effects with OXA against CRC, but the synergistic effect between Sal and ADM was not evident. The present study found that Sal induced generation of autophagy of HCT-
116 cells. Intervention of autophagy via 3-MA notably promoted synergistic effects between Sal and anti-tumor drugs including OXA, 5-FU and ADM. This finding suggests that Sal-induced autophagy probably impaired the synergistic effects with anti-tumor agents against CRC.

Autophagy is an important cellular process in response to various stress conditions to maintain proper cell function and homeostasis through the degradation of damaged intracellular proteins and organelles and the recycling of amino acids, free fatty acids or nucleotides derived from the degradation process $[7,9]$. Therefore, autophagy is 


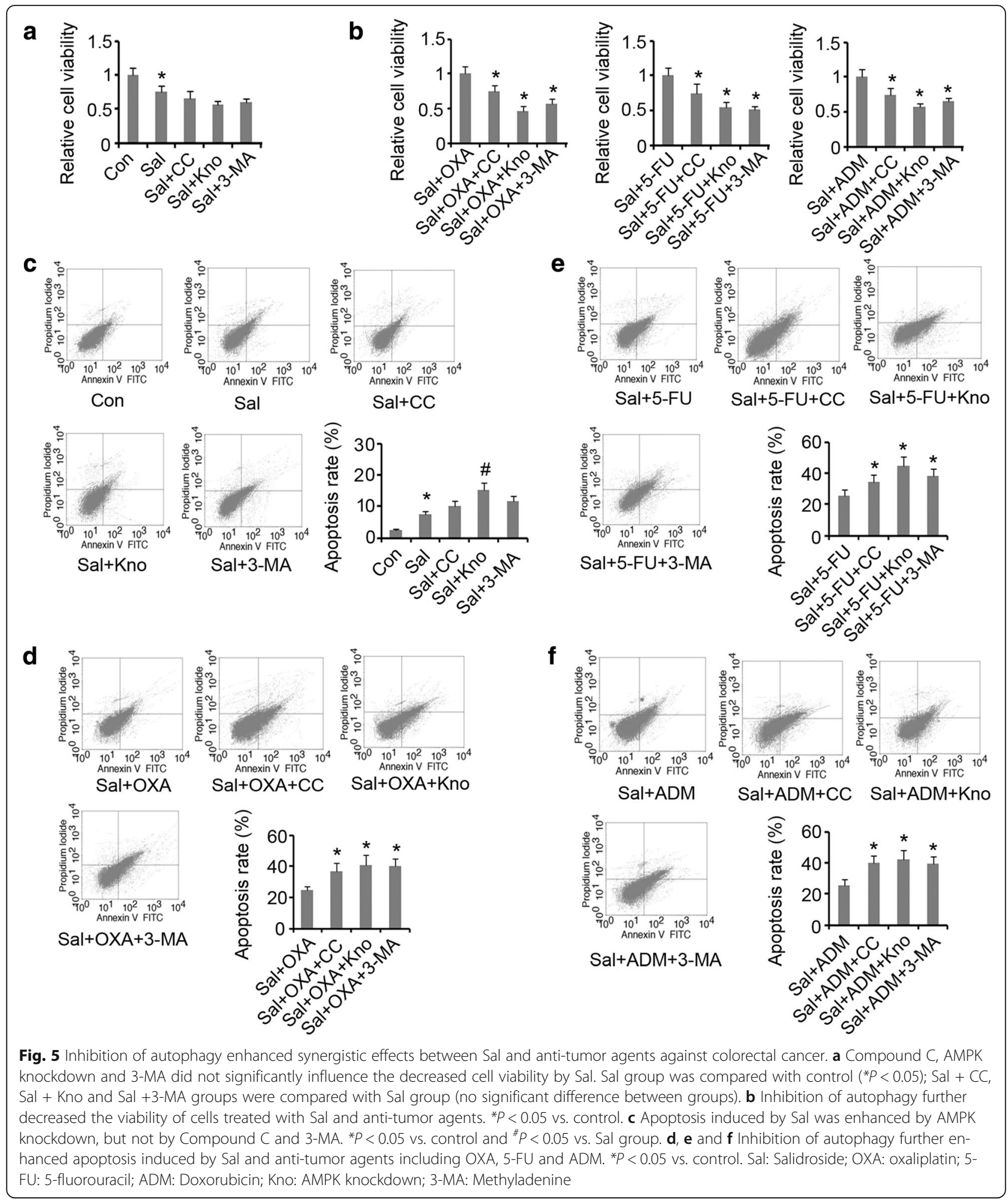

generally recognized as a cell pro-survival mechanism, although excessive induction of autophagy becomes an alternative mechanism for programmed cell death when exposing to overwhelming or persistent stress $[8,19]$. It has been suggested that targeting cytoprotective autophagy is a critical strategy to improve the anticancer activity of therapeutics [8]. Previous literature has demonstrated that sorafenib is able to activate autophagy, which confers a survival advantage to cancer cells and leads to sorafenib resistance. However, inhibiting autophagy 
promotes sorafenib-induced cell death and increase its anticancer effect [20]. In addition, inhibition of autophagy facilitates sensitivity of gefitinib and salinomycin in breast cancer and prostate cancer respectively $[8,21]$.

Autophagy is easily induced when cancer cells are exposed to some plant extracts, although the underlying mechanism is largely unknown. Autophagy is regulated by various signaling pathway, such as AMPK/mTOR, NF- $\kappa$ B, TGF- $\beta$ and JAK2/STAT3 [10-13]. To shed light on molecular basis that mediates Sal-induced autophagy, this study examined their expression profiles following Sal induction. Except for the up-regulation of p-AMPK, p-mTOR, p-NF- - B (p65), TGF- $\beta$, p-JAK2 and p-STAT3 were down-regulated by Sal. Previous literature has represented that autophagy is positively correlated with the activation of AMPK/mTOR, NF- $\mathrm{BB}$, TGF $\beta 1$ and JAK2/ STAT3 cascades [10-13], thus Sal-induced autophagy may associate AMPK signaling. To verify this hypothesis, this study blocked AMPK signaling via adding the specific inhibitor Compound $\mathrm{C}$ and transfecting siRNAAMPK. Both Compound $\mathrm{C}$ and AMPK knockdown reversed up-regulation of p-AMPK induced by Sal. The blockage of AMPK signaling is accompanied with reduced LC3B expression, suggesting that autophagy is inhibited. Therefore, it is suggested that AMPK signaling mediates autophagy of CRC cells induced by Sal.
The molecular mechanism by which AMPK regulates autophagy has been revealed in previous reports. AMPK regulating autophagy is dependent or independent of mTOR, through AMPK-mTOR-S6 K and AMPK-ULK1 signaling pathways, respectively $[22,23]$. mTOR activity is negatively modulated by AMPK, but mTOR inhibition associates improved autophagy, that is the reason why mTOR selective inhibitor Rapamycin is usually used as autophagy agonist. Although the present study demonstrated that Sal-induced autophagy is dependant of AMPK signaling, the mechanism by which Sal activates AMPK is unclear. Previous reports have suggested that plant extracts disturbing intracellular anti-/oxidative homeostasis is likely an important reason for AMPK activation $[6,8,9]$. Oxidative species have been confirmed to participate in the regulation of cell signaling. Further study should be warranted to elucidate mechanism by which Sal activates AMPK.

Previous study has demonstrated that Sal exerts antiproliferative and pro-apoptotic effects against cancer cells [18]. However, the present study found that Sal also triggered autophagy of CRC cells, which counteracts the anti-tumor effects of Sal alone or in combination with anti-tumor agents (as shown in Fig. 6a). Treatment with Sal up-regulated $\mathrm{p}$-AMPK, but down-regulated $\mathrm{p}$ mTOR, p-NF- $\mathrm{kB}$ (p65), TGF- $\beta$, p-JAK2 and p-STAT3 in

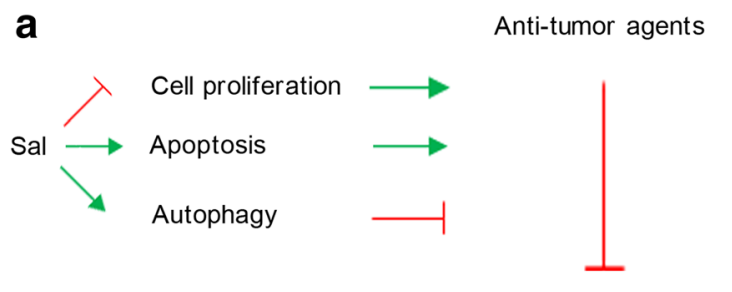

Colorectal cancer cells

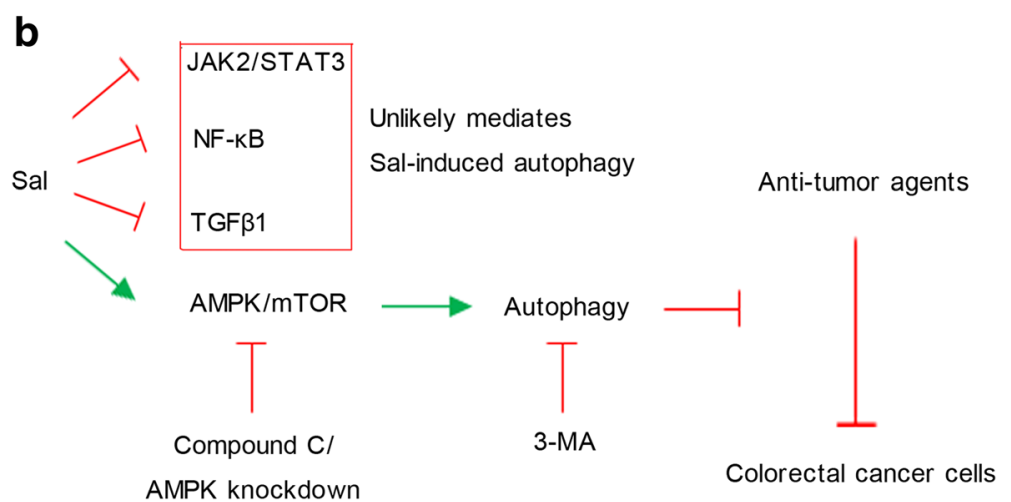

Fig. 6 Autophagy impairs synergistic effects of Sal and anti-tumor agents against CRC. a Although Sal exerts anti-proliferative and pro-apoptotic effects, it triggered autophagy of CRC cells, which counteracts the anti-tumor effects of Sal alone or in combination with anti-tumor agents. $\mathbf{b}$ Sal up-regulated p-AMPK, but down-regulated p-mTOR, p-NF-KB (p65), TGF- $\beta$, p-JAK2 and p-STAT3 in CRC cells. It was demonstrated that autophagy induced by Sal mainly associates with AMPK activation. Blockage of AMPK signaling via compound $\mathrm{C}$ and AMPK knockdown or direct inhibition of autophagy via 3-MA increased effectiveness of combined use of Sal with anti-tumor agents against CRC, which suggests that autophagy impairs synergistic effects of Sal and anti-tumor agents 
CRC cells. It was hypothesized that autophagy induced by Sal mainly associates with AMPK activation (as shown in Fig. 6b). This hypothesis was demonstrated by treatment with Compound $\mathrm{C}$ and by AMPK knockdown, as both of them inhibited the autophagy. The blockage of AMPK signaling or a direct inhibition of autophagy via 3-MA increased effectiveness of combined use of Sal with anti-tumor agents against CRC, which suggests that autophagy impairs synergistic effects of Sal and antitumor agents.

\section{Conclusions}

Inhibition of autophagy enhances synergistic effects of Sal and anti-tumor agents against colorectal cancer. This study provides experimental evidence and theoretical reference for improvement of a novel chemotherapy treatment protocol.

\section{Abbreviations}

5-FU: 5-fluorouracil; ADM: Doxorubicin; CRC: colorectal cancer; OXA: oxaliplatin; Sal: Salidroside

\section{Acknowledgements}

The authors thank Dr Chu-hong Xu (Department of clinical pharmacology, Union Hospital, Huazhong University of Science and Technology, Wuhan, China) for his assistance with analysis of the data.

\section{Funding}

This study was supported by the Shenzhen Municipal Commission of science and technology innovation (Grant No.JCYJ20150402152005631).

\section{Availability of data and materials}

All data supporting the study is presented in the manuscript or available upon request from the corresponding author of this manuscript (Hai Li) at Email: lihaiges@hust.edu.cn.

\section{Authors' contributions}

HL taken the responsibility of the experimental design. HL and CC performed the study and wrote the manuscript. Both authors have read and approved of the final manuscript.

\section{Ethics approval and consent to participate}

This information is not relevant.

\section{Consent for publication}

Not applicable.

\section{Competing interests}

The authors declare that they have no competing interests.

\section{Publisher's Note}

Springer Nature remains neutral with regard to jurisdictional claims in published maps and institutional affiliations.

\footnotetext{
Author details

'Department of Geriatrics, Tongji Hospital, Tongji Medical College, Huazhong University of Science and Technology, Wuhan, China. ${ }^{2}$ Department of Breast and Thyroid Surgery, Union Hospital, Tongji Medical College, Huazhong University of Science and Technology, Wuhan, China.

Received: 19 July 2017 Accepted: 6 December 2017 Published online: 16 December 2017

\section{References}

1. Sonowal H, Pal PB, Wen JJ, Awasthi S, Ramana KV, Srivastava SK. Aldose reductase inhibitor increases doxorubicin-sensitivity of colon cancer cells and decreases cardiotoxicity. Sci Rep. 2017;7:3182.
}

2. Huang WW, Hsieh KP, Huang RY, Yang YH. Role of cyclooxygenase-2 inhibitors in the survival outcome of colorectal cancer patients: a population-based cohort study. Kaohsiung J Med Sci. 2017;33:308-14.

3. Einbond LS, Negrin A, Kulakowski DM, Wu HA, Antonetti V, Jalees F, et al. Traditional preparations of kava (Piper Methysticum) inhibit the growth of human colon cancer cells in vitro. Phytomedicine. 2017;24:1-13.

4. Chen WT, Yang TS, Chen HC, Chen HH, Chiang HC, Lin TC, et al. Effectiveness of a novel herbal agent MB-6 as a potential adjunct to 5fluoracil-based chemotherapy in colorectal cancer. Nutr Res. 2014;34:585-94.

5. Xie J, Liu JH, Liu H, Liao XZ, Chen Y, Lin MG, et al. Tanshinone IIA combined with adriamycin inhibited malignant biological behaviors of NSCLC A549 cell line in a synergistic way. BMC Cancer. 2016;16:899.

6. Xu Z, Huang CM, Shao Z, Zhao XP, Wang M, Yan TL, et al. Autophagy induced by areca nut extract contributes to decreasing cisplatin toxicity in oral squamous cell carcinoma cells: roles of reactive oxygen species/AMPK signaling. Int J Mol Sci. 2017;18 doi:10.3390/ijms18030524.

7. Law BY, Mok SW, Wu AG, Lam CW, Yu MX, Wong VK. New potential pharmacological functions of Chinese herbal medicines via regulation of autophagy. Molecules. 2016;21(3):359.

8. Kim KY, Park KI, Kim SH, Yu SN, Park SG, Kim YW, et al. Inhibition of autophagy promotes salinomycin-induced apoptosis via reactive oxygen speciesmediated PI3K/AKT/mTOR and ERK/p38 MAPK-dependent signaling in human prostate cancer cells. Int J Mol Sci. 2017;18 doi:10.3390/ijms18051088.

9. Bastola T, An RB, Kim YC, Kim J, Seo J. Cearoin induces autophagy, ERK activation and apoptosis via ROS generation in SH-SY5Y neuroblastoma cells. Molecules. 2017:22 doi:10.3390/molecules22020242.

10. Hao M, Zhu S, Hu L, Zhu H, Wu X, Li Q. Myocardial ischemic postconditioning promotes autophagy against ischemia reperfusion injury via the activation of the nNOS/AMPK/mTOR Pathway. Int J Mol Sci. 2017;18 doi:10.3390/ijms18030614.

11. Zhou DZ, Sun HY, Yue JQ, Peng Y, Chen YM, Zhong ZJ. Dihydromyricetin induces apoptosis and cytoprotective autophagy through ROS-NF-KB signalling in human melanoma cells. Free Radic Res. 2017;51:517-28.

12. Shen J, Zhao DS, Li MZ. TGF- $\beta 1$ promotes human gastric carcinoma SGC7901 cells invasion by inducing autophagy. Eur Rev Med Pharmacol Sci. 2017;21:1013-9.

13. Pratt J, Annabi B. Induction of autophagy biomarker BNIP3 requires a JAK2/ STAT3 and MT1-MMP signaling interplay in Concanavalin-A-activated U87 glioblastoma cells. Cell Signal. 2014;26:917-24.

14. Zhao G, Shi A, Fan Z, Du Y. Salidroside inhibits the growth of human breast cancer in vitro and in vivo. Oncol Rep. 2015;33:2553-60.

15. Wang J, Li JZ, Lu AX, Zhang KF, Li BJ. Anticancer effect of salidroside on A549 lung cancer cells through inhibition of oxidative stress and phosphop38 expression. Oncol Lett. 2014;7:1159-64.

16. Lv C, Huang Y, Liu ZX, Yu D, Bai ZM. Salidroside reduces renal cell carcinoma proliferation by inhibiting JAK2/STAT3 signaling. Cancer Biomark. 2016;17:41-7.

17. Zhu Q, Li H, Liu Y, Jiang L. Knockdown of CFTR enhances sensitivity of prostate cancer cells to cisplatin via inhibition of autophagy. Neoplasma. 2017:64:709-17.

18. Ma YG, Wang JW, Bai YG, Liu M, Xie MJ, Dai ZJ. Salidroside contributes to reducing blood pressure and alleviating cerebrovascular contractile activity in diabetic Goto-Kakizaki rats by inhibition of L-type calcium channel in smooth muscle cells. BMC Pharmacol Toxicol. 2017;18:30.

19. Chan SH, Kikkawa U, Matsuzaki H, Chen JH, Chang WC. Insulin receptor substrate-1 prevents autophagy-dependent cell death caused by oxidative stress in mouse NIH/3T3 cells. J Biomed Sci. 2012;19:64.

20. Rong $L W$, Wang $R X$, Zheng $X L$, Feng $X Q$, Zhang $L$, Zhang $L$, et al. Combination of wogonin and sorafenib effectively kills human hepatocellular carcinoma cells through apoptosis potentiation and autophagy inhibition. Oncol Lett. 2017;13:5028-34.

21. Liu Z, He K, Ma Q, Yu Q, Liu C, Ndege I, et al. Autophagy inhibitor facilitates gefitinib sensitivity in vitro and in vivo by activating mitochondrial apoptosis in triple negative breast cancer. PLoS One. 2017;12:e0177694.

22. Jang JE, Eom Jl, Jeung HK, Cheong JW, Lee JY, Kim JS, et al. Targeting AMPK-ULK1-mediated autophagy for combating BET inhibitor resistance in acute myeloid leukemia stem cells. Autophagy. 2017;13:761-2.

23. Cho YS, Yen CN, Shim JS, Kang DH, Kang SW, Liu JO, et al. Antidepressant indatraline induces autophagy and inhibits restenosis via suppression of mTOR/S6 kinase signaling pathway. Sci Rep. 2016;6:34655. 